Supporting Information

\title{
Cyanide-Bridged Mn(III)-Fe(III) Bimetallic Complexes Based on the Pentacyano(1-methylimidazole)ferrate(III) Building Block: Structure and Magnetic Characterizations
}

\author{
Wei-Wei Ni, Zhong-Hai Ni, Ai-Li Cui, Xin Liang, Hui-Zhong Kou* \\ Department of Chemistry, Tsinghua University, Beijing 100084, P. R. China \\ *E-mail: kouhz@mail.tsinghua.edu.cn. Fax: 86-10-62771748
}


Table S1. Selected Bond Distances (Å) and Angles (deg) of Complex 3.

\begin{tabular}{|c|c|c|c|}
\hline $\mathrm{Fe}(1)-\mathrm{C}(1)$ & $1.946(6)$ & $\mathrm{Fe}(1)-\mathrm{C}(2)$ & $1.920(6)$ \\
\hline $\mathrm{Fe}(1)-\mathrm{C}(3)$ & $1.921(7)$ & $\mathrm{Fe}(1)-\mathrm{C}(4)$ & $1.944(6)$ \\
\hline $\mathrm{Fe}(1)-\mathrm{C}(5)$ & $1.945(7)$ & $\mathrm{Fe}(1)-\mathrm{N}(6)$ & $2.010(6)$ \\
\hline $\mathrm{Fe}(2)-\mathrm{C}(43)$ & $1.921(7)$ & $\mathrm{Fe}(2)-\mathrm{C}(44)$ & $1.937(6)$ \\
\hline $\mathrm{Fe}(2)-\mathrm{C}(45)$ & $1.969(6)$ & $\mathrm{Fe}(2)-\mathrm{C}(46)$ & $1.956(6)$ \\
\hline $\mathrm{Fe}(2)-\mathrm{C}(47)$ & $1.947(6)$ & $\mathrm{Fe}(2)-\mathrm{N}(16)$ & $1.990(5)$ \\
\hline $\mathrm{Mn}(1)-\mathrm{N}(1)$ & $2.221(5)$ & $\mathrm{Mn}(1)-\mathrm{O}(1 \mathrm{~W})$ & $2.246(4)$ \\
\hline $\mathrm{Mn}(1)-\mathrm{O}(1)$ & $1.908(4)$ & $\operatorname{Mn}(1)-N(8)$ & $2.098(5)$ \\
\hline $\mathrm{Mn}(1)-\mathrm{O}(2)$ & $1.907(4)$ & $\operatorname{Mn}(1)-\mathrm{N}(9)$ & $2.042(5)$ \\
\hline $\operatorname{Mn}(2)-\mathrm{N}(2)$ & $2.249(5)$ & $\mathrm{Mn}(2)-\mathrm{O}(2 \mathrm{~W})$ & $2.233(4)$ \\
\hline $\mathrm{Mn}(2)-\mathrm{O}(3)$ & $1.883(4)$ & $\operatorname{Mn}(2)-N(10)$ & $2.051(5)$ \\
\hline $\mathrm{Mn}(2)-\mathrm{O}(4)$ & $1.918(4)$ & $\operatorname{Mn}(2)-\mathrm{N}(11)$ & $2.059(5)$ \\
\hline $\operatorname{Mn}(3)-N(13)$ & $2.229(5)$ & $\mathrm{Mn}(3)-\mathrm{O}(3 \mathrm{~W})$ & $2.234(4)$ \\
\hline $\operatorname{Mn}(3)-\mathrm{O}(5)$ & $1.907(4)$ & $\operatorname{Mn}(3)-N(18)$ & $2.049(5)$ \\
\hline $\mathrm{Mn}(3)-\mathrm{O}(6)$ & $1.907(4)$ & $\operatorname{Mn}(3)-N(19)$ & $2.070(5)$ \\
\hline $\operatorname{Mn}(4)-N(12)$ & $2.298(5)$ & $\mathrm{Mn}(4)-\mathrm{O}(4 \mathrm{~W})$ & $2.222(4)$ \\
\hline $\mathrm{Mn}(4)-\mathrm{O}(7)$ & $1.875(4)$ & $\operatorname{Mn}(4)-\mathrm{N}(20)$ & $2.029(5)$ \\
\hline $\mathrm{Mn}(4)-\mathrm{O}(8)$ & $1.892(4)$ & $\operatorname{Mn}(4)-N(21)$ & $2.050(5)$ \\
\hline $\mathrm{Mn}(1)-\mathrm{N}(1)-\mathrm{C}(1)$ & $165.2(6)$ & $\mathrm{N}(1)-\mathrm{C}(1)-\mathrm{Fe}(1)$ & $176.8(6)$ \\
\hline $\operatorname{Mn}(2)-N(2)-C(2)$ & $156.9(5)$ & $\mathrm{N}(2)-\mathrm{C}(2)-\mathrm{Fe}(1)$ & $174.8(6)$ \\
\hline $\mathrm{Mn}(3)-\mathrm{N}(13)-\mathrm{C}(46)$ & $152.8(5)$ & $\mathrm{N}(13)-\mathrm{C}(46)-\mathrm{Fe}(2)$ & $174.5(6)$ \\
\hline $\mathrm{Mn}(4)-\mathrm{N}(12)-\mathrm{C}(44)$ & $147.8(5)$ & $\mathrm{N}(12)-\mathrm{C}(44)-\mathrm{Fe}(2)$ & $177.1(6)$ \\
\hline
\end{tabular}




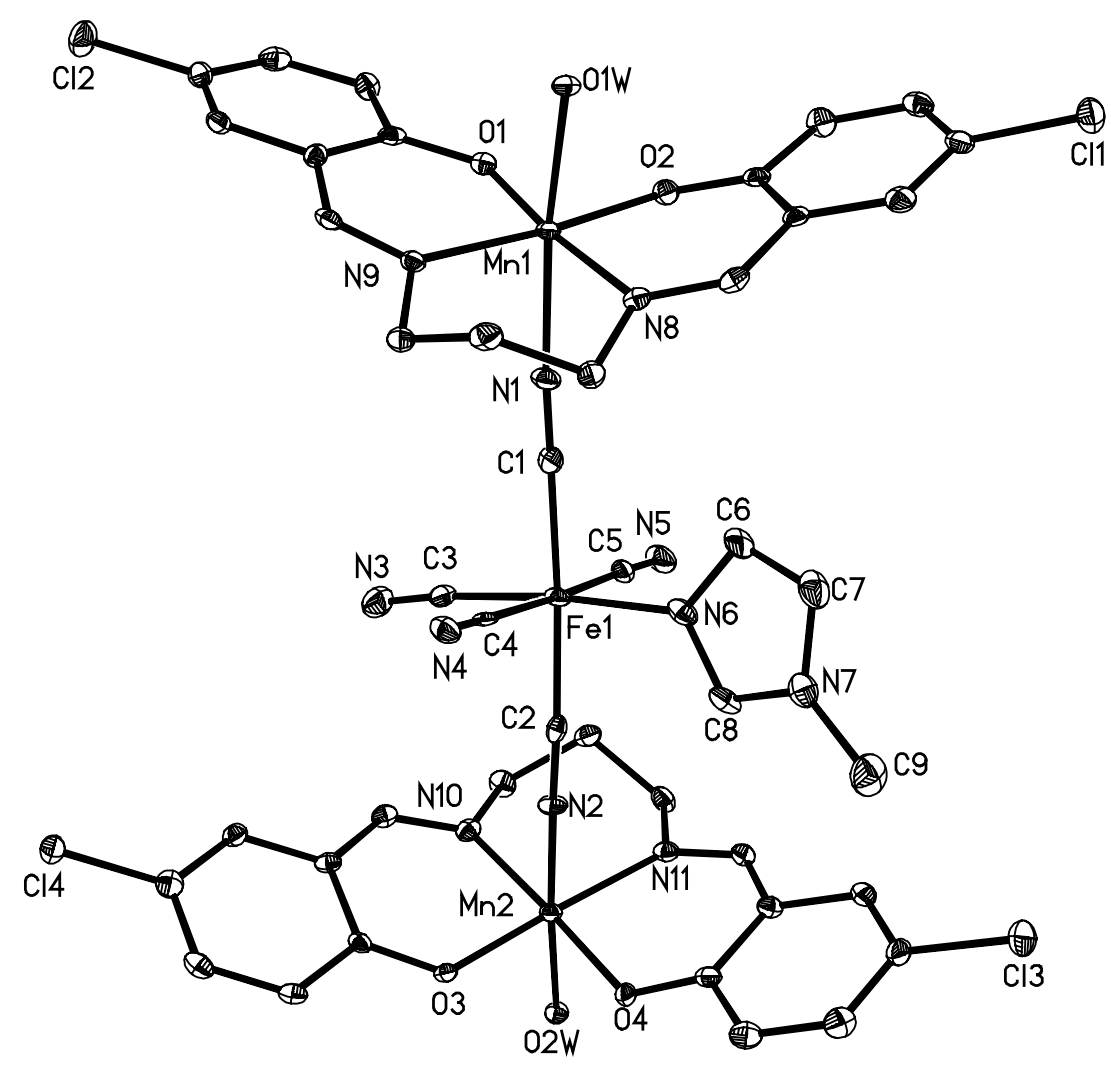

Figure S1. One of the two independent trans-trinuclear units in complex $\mathbf{3}$ with atom labeling scheme showing $30 \%$ probability thermal ellipsoids (hydrogen atoms are omitted for clarity). 


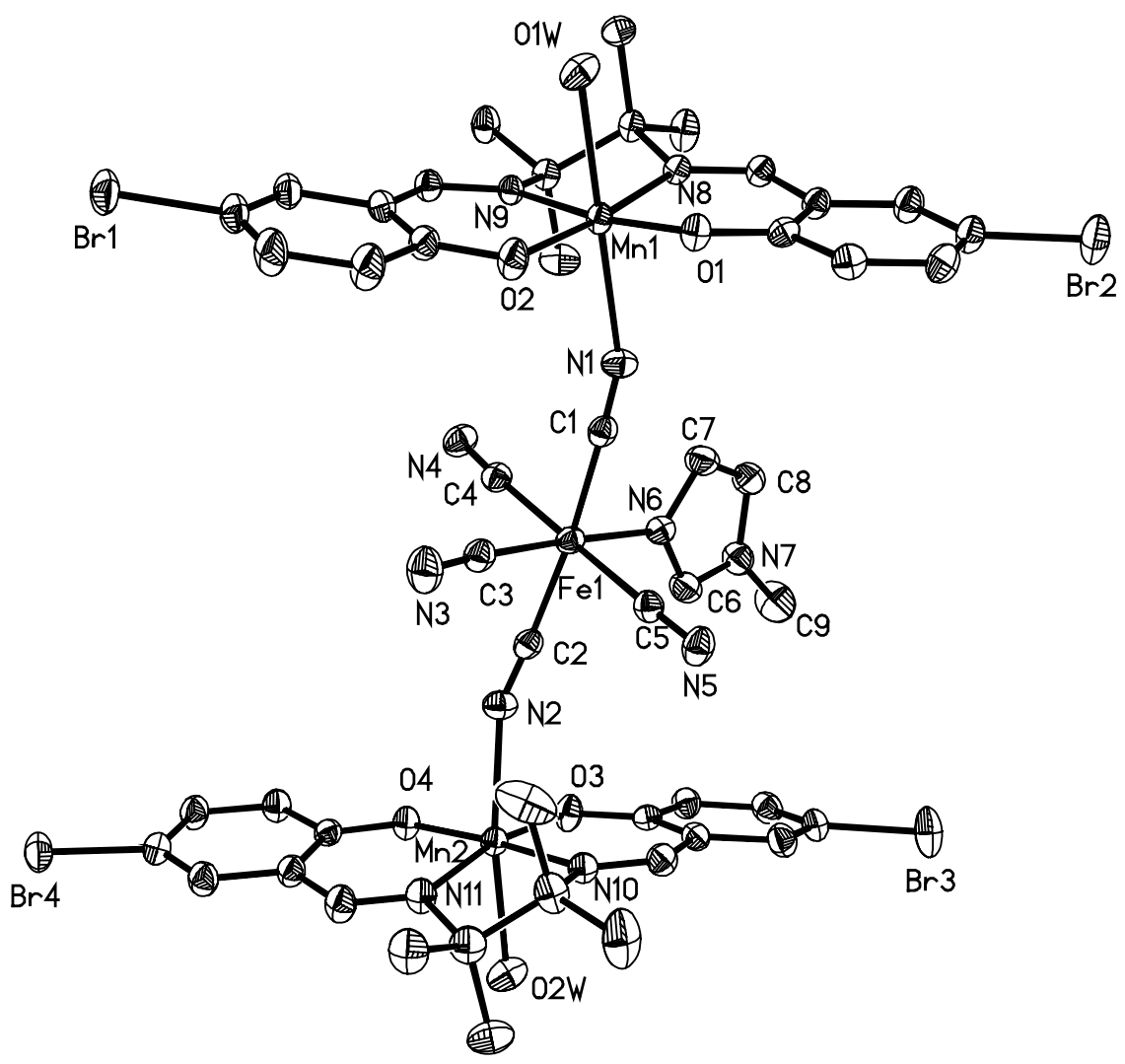

Figure S2. Trinuclear structure of complex $\mathbf{5}$ with atom labeling scheme showing $30 \%$ probability thermal ellipsoids (hydrogen atoms and crystallized methanol molecules are omitted for clarity). 


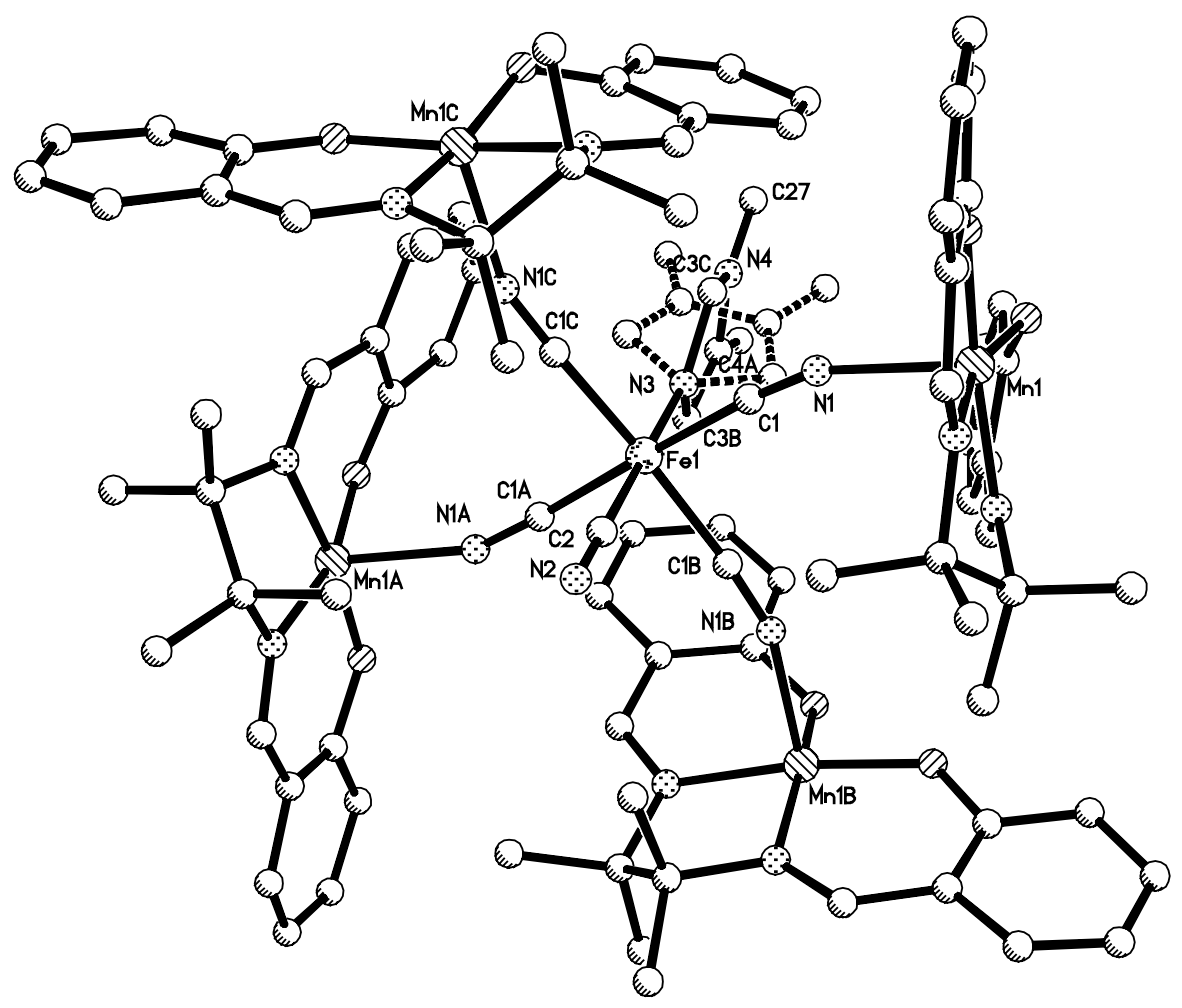

Figure S3. Structure of complex 7 (hydrogen atoms, perchlorate ions and water molecules are omitted for clarity). Dashed lines represent the disordered 1-methylimidazole groups. Selected bond distances $(\AA)$ and angles (deg): Fe(1)-C(1) 1.936(8); $\quad \mathrm{Fe}(1)-\mathrm{C}(2) \quad 1.935(14) ; \quad \mathrm{Fe}(1)-\mathrm{N}(3) \quad 1.961(13) ; \quad \mathrm{Mn}(1)-\mathrm{N}(1) \quad 2.197(8) ;$ $\mathrm{Mn}(1)-\mathrm{O}(1) \quad 1.871(5) ; \quad \mathrm{Mn}(1)-\mathrm{O}(2) \quad 1.863(6) ; \quad \mathrm{Mn}(1)-\mathrm{N}(5) \quad 1.984(8) ; \quad \mathrm{Mn}(1)-\mathrm{N}(6)$ 1.969(7); $\mathrm{Fe}(1)-\mathrm{C}(1)-\mathrm{N}(1)$ 176.0(7); C(1)-N(1)-Mn(1) 157.3(7). 
Eq. 1:

$$
\chi_{\mathrm{t}}=\frac{N g^{2} \beta^{2}}{k T}\left[\frac{\mathrm{A}}{\mathrm{B}}\right]
$$

in which $\mathrm{A}=165+84 \exp (-9 J / k T)+84 \exp (-J / k T)+35 \exp (-8 J / k T)+35 \exp (-2 J / k T)$

$+10 \exp (-7 J / k T)+10 \exp (-3 J / k T)+\exp (-6 J / k T)+\exp (-4 J / k T)$ and $\mathrm{B}=20+$ $16 \exp (-9 J / k T)+16 \exp (-J / k T)+12 \exp (-8 J / k T)+12 \exp (-2 J / k T)+8 \exp (-7 J / k T)+$ $8 \exp (-3 J / k T)+4 \exp (-6 J / k T)+4 \exp (-4 J / k T)$.

Eq. 2:

$$
\chi_{\mathrm{m}}=\frac{\chi_{\mathrm{t}}}{1-\chi_{\mathrm{t}}\left(2 z J^{\prime} / N g^{2} \beta^{2}\right)}
$$

Eq. 3:

$$
\chi_{\mathrm{m}}=\frac{N g^{2} \beta^{2}}{k T}\left[\frac{\mathrm{A}}{\mathrm{B}}\right]
$$

in which $\mathrm{A}=1+9 \exp (2 D / k T)+25 \exp (6 D / k T)+49 \exp (12 D / k T)+81 \exp (20 D / k T)+$ $84 \exp (-9 J / k T)+84 \exp (-J / k T)+35 \exp (-8 J / k T)+35 \exp (-2 J / k T)+10 \exp (-7 J / k T)+$ $10 \exp (-3 J / k T)+\exp (-6 J / k T)+\exp (-4 J / k T)$ and $\mathrm{B}=4+4 \exp (2 D / k T)+4 \exp (6 D / k T)+$ $4 \exp (12 D / k T)+4 \exp (20 D / k T)+16 \exp (-9 J / k T)+16 \exp (-J / k T)+12 \exp (-8 J / k T)+$ $12 \exp (-2 J / k T)+8 \exp (-7 J / k T)+8 \exp (-3 J / k T)+4 \exp (-6 J / k T)+4 \exp (-4 J / k T)$. 

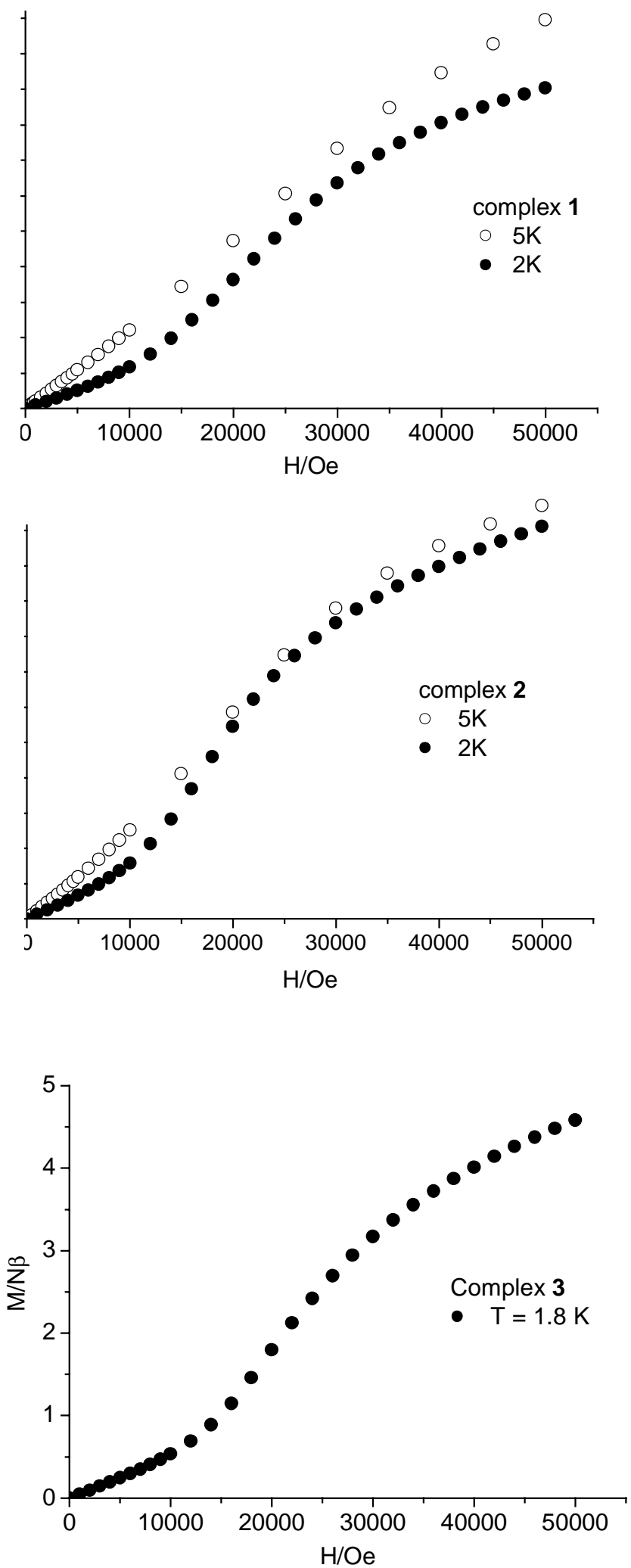

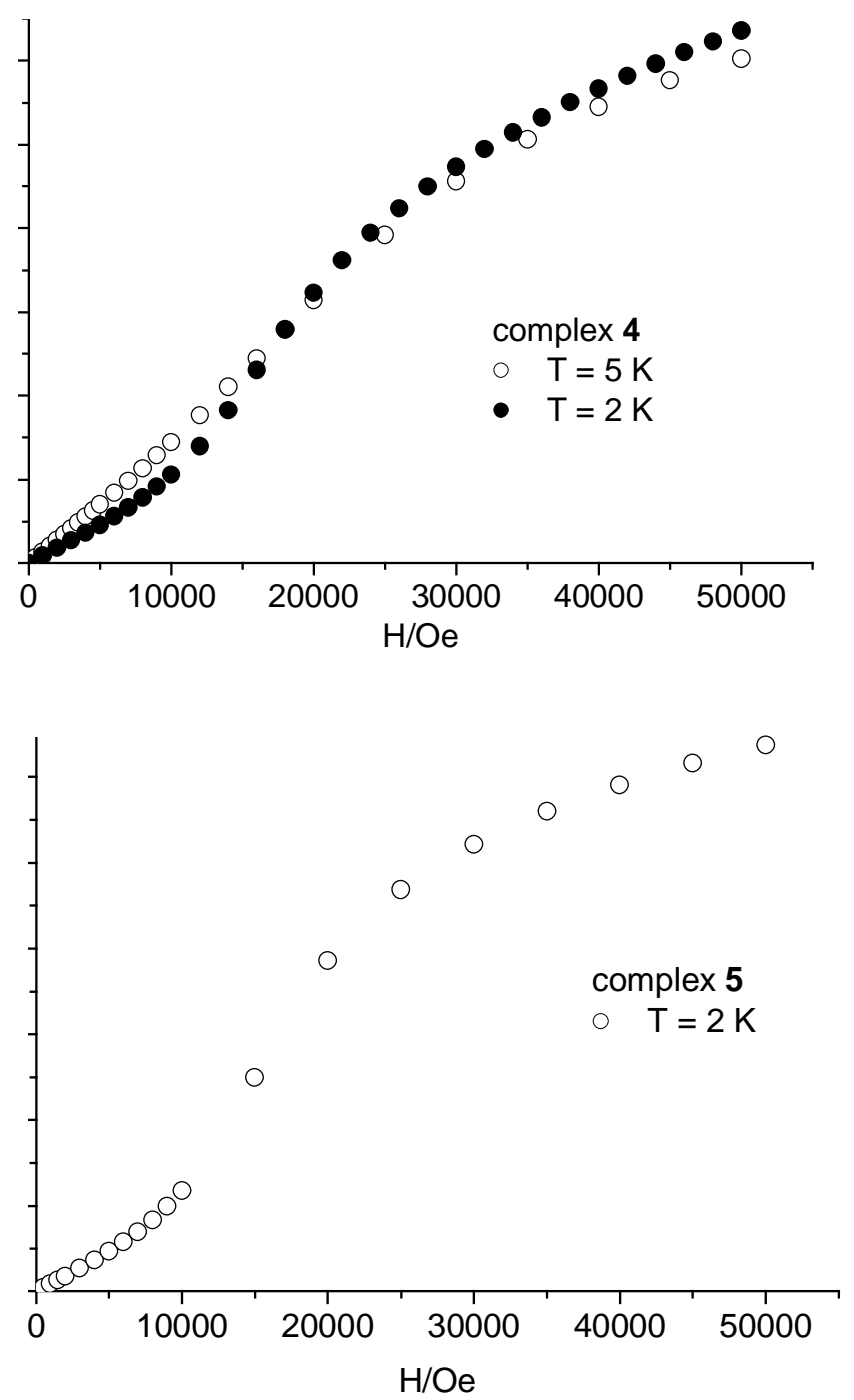

Figure S4. Field dependence of magnetization for the trinuclear complexes. 


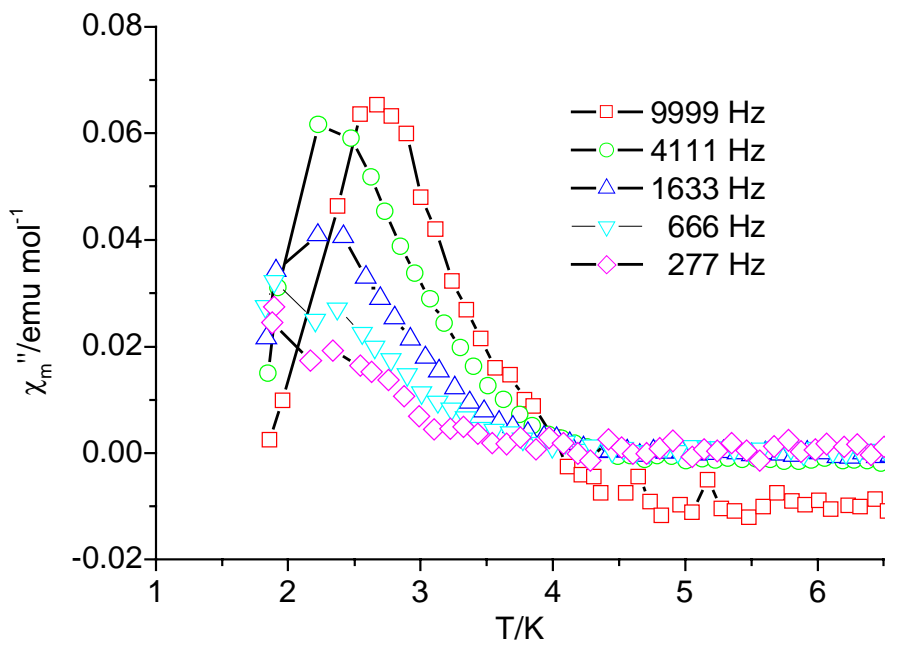

Figure S5. Out-of-phase component of the zero-static $\left(\mathrm{H}_{\mathrm{DC}}=0\right) \mathrm{AC}$ susceptibility at different frequencies for $\mathbf{5}$. 